\title{
Correction to: Soilless tomato grown under nutritional stress increases green biomass but not yield or quality in presence of biochar as growing medium
}

\author{
Daniele Massa ${ }^{1}(1) \cdot$ Alessandra Bonetti $^{2} \cdot$ Sonia Cacini ${ }^{1} \cdot$ Cecilia Faraloni $^{3} \cdot$ Domenico Prisa $^{1} \cdot$ Lorenza Tuccio $^{4}$. \\ Raffaella Petruccelli ${ }^{3}$
}

Published online: 29 October 2019

(c) Korean Society for Horticultural Science 2019

\section{Correction to: \\ Horticulture, Environment, and Biotechnology \\ https://doi.org/10.1007/s13580-019-00169-x}

The original version of this article unfortunately contained a mistake. The first and last names of all authors were interchanged; the corrected author list is given above. The original article has been corrected.

Publisher's Note Springer Nature remains neutral with regard to jurisdictional claims in published maps and institutional affiliations.

The original article can be found online at https://doi.org/10.1007/ s13580-019-00169-x.

Daniele Massa

daniele.massa@crea.gov.it

1 CREA Research Centre for Vegetable and Ornamental Crops, Council for Agricultural Research and Economics, Via dei Fiori 8, 51017 Pescia, PT, Italy

2 CNR-IRET Istituto di Ricerca sugli Ecositemi Terrestri, Consiglio Nazionale delle Ricerche, Via Madonna del Piano 10, 50019 Sesto Fiorentino, FI, Italy

3 CNR-IVALSA Istituto per la Valorizzazione del Legno e delle Specie Arboree, Consiglio Nazionale delle Ricerche, Via Madonna del Piano 10, 50019 Sesto Fiorentino, FI, Italy

4 CNR-IFAC Istituto di Fisica Applicata "Nello Carraro", Consiglio Nazionale delle Ricerche, Via Madonna del Piano 10, 50019 Sesto Fiorentino, FI, Italy 